\title{
Microplastic release from the degradation of polypropylene feeding bottles during infant formula preparation
}

\author{
Dunzhu Li, ${ }^{1,2}$, Yunhong Shi² $\mathbf{2}$, Luming Yang ${ }^{2,1}$, Liwen Xiao ${ }^{2,3 *}$, Daniel K. Kehoe', Yurii K. \\ Gun'ko ${ }^{4,5}$, John J. Boland ${ }^{1,4 *}$, Jing Jing Wang ${ }^{1 *}$
}

\begin{abstract}
${ }^{1}$ AMBER Research Centre and Centre for Research on Adaptive Nanostructures and Nanodevices (CRANN), Trinity College Dublin, D2, Ireland

${ }^{2}$ Department of Civil, Structural and Environmental Engineering, Trinity College Dublin, Dublin 2, Ireland

${ }^{3}$ TrinityHaus, Trinity College Dublin, Dublin 2, Ireland

${ }^{4}$ School of Chemistry, Trinity College Dublin, Dublin 2, Ireland

${ }^{5}$ BEACON, Bioeconomy SFI Research Centre, University College Dublin, Dublin 4, Ireland

*Correspondence to: Jing Jing Wang, jiwang@tcd.ie; Liwen Xiao, liwen.xiao@tcd.ie;John J. Boland, jboland@tcd.ie

tEqual contributions
\end{abstract}

\begin{abstract}
:
Polypropylene-based products are commonly used for food preparation and storage, but their capacity to release microplastics is poorly understood. We investigated the potential exposure of infants to microplastics from consuming formula prepared in polypropylene infant feeding bottles (PP-IFBs). Here, we show that PP-IFBs release microplastics with values as high as $16,200,000$ particles/L. Scenario studies showed that PP-IFB sterilisation and exposure to high temperature water significantly increase microplastic release. A 21-day test of PP-IFBs showed periodic fluctuations in microplastic release. To estimate the potential global exposure to infants up to 12 months old, we surveyed 48 regions finding values ranging from 14,600 to 4,550,000 particles per capita per day, depending on the region. We demonstrate that infant exposure to microplastics is higher than previously recognized due to the prevalence of polypropylene-based products in formula preparation, highlighting an urgent need to assess whether MPs exposure at these levels pose a risk to infant health. A formula preparation scheme that reduces the exposure of infants to microplastics is suggested.
\end{abstract}


Microplastics (MPs) are a global concern ${ }^{1-5}$ due to their potential risk to human health ${ }^{6-9}$. Estimated MPs consumption via food chain and inhalation pathways ranges from 74,000 to 211,000 particles annually in the US ${ }^{10}$; MPs have been detected in human stool ${ }^{11}$. Exposure to MPs can induce gut microbiota dysbiosis and lipid metabolism disorder in mice ${ }^{12,13}$; submicron MPs can penetrate the fish blood-brain barrier, inducing brain damage and behavioural disorders ${ }^{14}$

Research on MPs has focused on major food and water sources (e.g. fish) and the potential transfer of MPs from the ocean to humans through the food chain ${ }^{10}$, but little is known about the direct release of MPs from plastic products. Two specific products with plastic containers (polyethylene terephthalate-PET water bottles and PET/nylon teabags ${ }^{15-18}$ ) were found to release high levels of MPs under daily use conditions. Polypropylene (PP) MPs accounted for $62.8 \%$ of all MPs detected in adult stool, but the origin of these MPs is unknown ${ }^{11}$. The annual production of PP accounts for about $20 \%$ of all non-fiber plastics production ${ }^{19}$ and is considered to be a safe plastic that is suitable for many applications ${ }^{20}$, such that PP is the most widely used plastic in food preparation ${ }^{21}$.

Polypropylene infant-feeding bottles (PP-IFBs) are widely used for the preparation of infant formula. IFBs are routinely exposed to high temperature water and endure shaking during formula preparation procedures. Since mechanical friction force can break down PP into MPs ${ }^{22-25}$, there is the potential for MP release from PP-IFBs. To assess the prevalence of PPIFBs, we mined IFB sales data from a local leading e-commerce sites (data from each Amazon site were mined using the Jungle Scout platform while the data from non-Amazon sites were directly collected via the site's web page) from 48 regions, covering $77.6 \%$ of the global population (Fig. 1). We identified that PP-IFBs in the form of PP bottles, PP accessories, or both (Fig. 1a) account for $82.5 \%$ of the global IFB market, with regional differences in the preference for PP-IFBs, accessories and non-PP products. The balance of the market share is dominated by glass IFBs. Next, we estimated infant MPs exposure by developing a protocol to quantify and characterize the PP-MPs release from PP-IFBs using ten representative PPIFBs products, accounting for $68.8 \%$ of the global market (Fig. 1b). The effect of water temperature, sterilisation and repeated use over 21-day period on the levels of MP release were assessed. Combining the MP release data from PP-IFBs with local non-breast-feeding rates and milk intake volumes, we estimated the exposure of 12-month-old infants to MPs in 48 regions.

\section{Results}

Sample preparation and measurement protocol. We tested the quantity of MPs released from PP-IFBs during standard formula-preparation steps involving WHOrecommended ${ }^{26}$ cleaning, sterilising, and mixing techniques (Fig. 2a). After thoroughly cleaning each of the brand-new PP-IFBs (Product (Pd) 1-10), we soaked them in $95{ }^{\circ} \mathrm{C}$ deionized (DI) water for 5 minutes (cleaning and sterilising). After air drying, we poured $70^{\circ} \mathrm{C}$ DI water (the recommended temperature for infant formula preparation ${ }^{26}$ ) into the IFBs, which were then mechanically shaken for 60 s to simulate the formula mixing process (180 $\mathrm{rpm}$ in a reciprocating shaker). After cooling, we filtered the water samples from the PP-IFBs using a gold-coated filter with pore size of $0.8 \mu \mathrm{m}$. The quantity and topography of the released PP-MPs were determined by Raman spectroscopy and atomic force microscopy 
(AFM), (see Fig. 2b-e, and details in Supplementary Figures 2-3 and Notes 2, 3 and 6). To confirm the reliability of this protocol (Supplementary Figures 4-6 and Note 4), we first conducted a recovery test employing standard Polystyrene microplastic samples (PS-MPs, 64050-15, Polysciences) of similar size and concentration to the MPs released from the PP products. Using the same protocol, we confirmed a $94.9 \pm 0.4 \%$ ( \pm represents standard error of the mean value) recovery of the polystyrene MPs, which is comparable to a previous study on $\mathrm{MPs}^{27}$. In addition, a third-party (Keyence, test using VHX-7000 equipped with an auto focus, Fig. 2f) undertook a blind test to further check the reliability of our methods. The PPMPs results from our lab showed a similar mean value and range to those from the third party $(4,000,000 \pm 590,000$ versus $4,300,000 \pm 360,000$ particles/L, Fig. $2 f)$. We also conducted a two-sided $t$-test (OriginPro 8.6) and found no significant difference $(P>0.05)$ between the data sets. These results confirmed the reliability of the preparation and analyses methods developed in this study.

Since real-world formula preparation involves the PP-IFBs coming into direct contact with infant formula and tap water, we developed control experiments to test the impact of using tap-water, DI water and infant formula on PP-MP release levels from IFBs and found that using DI water, tap water or formula does not significantly affect the MPs release from PP products. (Fig. 2g-i, and details in Supplementary Note 5). Hence, DI water was used throughout this study to reduce the usage of gold-coated filters and to simplify sample preparation.

MPs release from PP-IFBs during formula preparation. The release characteristics of the 10 representative PP-IFBs products were measured. IFB products 1-8, which have both PP bottle bodies and PP accessories, released high quantities of PP-MPs: from 1,310,000 \pm 130,000 to $16,200,000 \pm 1,300,000$ particles/L (Fig. 3a; Supplementary Table 1). IFB products 9-10, which only have PP accessories (gravity ball and round disk, Fig. 3a and Supplementary Table 1), released $69,700 \pm 9,800$ and $267,000 \pm 15,000$ particles/L, respectively. These results contrast strongly with the WHO assessment of the low risk of exposure to MPs in drinking water (mean values ranged from $10^{-3}$ to $10^{3}$ particles $/ \mathrm{L}^{15}$ ). Depending on the IFB product, the measured MP levels are 3 to 5 orders of magnitude higher than the background level collected using the control sample (170 \pm 54 particles/L, Fig. 3a), confirming that the PP-IFBs are the main source of measured MPs. We chose Pd 1-3 for a more detailed analysis as they collectively represent over $43 \%$ of the global PP-IFB market (Supplementary Table 1 ).

Optical microscopy was used to determine the size of MPs while AFM was used to measure their surface topography and thickness. The majority of MPs were smaller than $20 \mu \mathrm{m}$ (Supplementary Figure 2). The detailed size distribution for one product is shown in Figure 3b. Typical AFM images (Fig. 3c-k) reveal that the majority of MPs are flake-like with a coarse surface with an average thickness around one-tenth of the lateral dimensions. Some released MPs exhibit a layered morphology (Fig. 3i-k), consistent with the known structure of $\mathrm{PP}^{28}$.

The influence of different preparation conditions on MP release. Plastics are thermally-sensitive materials $\mathrm{s}^{20}$ and repeated use under high temperature conditions may accelerate degradation and MP release. During infant formula preparation, IFBs are exposed 
to temperatures up to $100^{\circ} \mathrm{C}$, in accordance with the WHO guidelines ${ }^{26}$. To assess the influence of temperature on MP release, we exposed PP-IFBs to DI water with temperatures of $25^{\circ} \mathrm{C}, 40^{\circ} \mathrm{C}, 70^{\circ} \mathrm{C}$ and $95^{\circ} \mathrm{C}$. Figure 4 a shows that the MP release from PP-IFBs is affected by temperature. For Pd1, there was about two orders of magnitude increase in MP release, from 0.6 million particles/L to 55 million particles/L when the temperature was increased from 25 to $95{ }^{\circ} \mathrm{C}$. Mechanical shaking at $25^{\circ} \mathrm{C}$ resulted in substantial MP release. We found similar but weaker MP release patterns for the other IFB products (Fig. 4a). A robust powerlaw formula ( $\mathrm{R}^{2}$ range of $0.8-0.98$ ) can be used to predict the potential release of PP-MPs across the temperature range $25^{\circ} \mathrm{C}$ to $95^{\circ} \mathrm{C}$. Since $100^{\circ} \mathrm{C}$ sterilisation is a standard cleaning procedure for IFBs (a WHO recommendation ${ }^{26}$ ), we also compared the MPs released from IFBs with and without sterilisation (Supplementary Note 2.3). We found that the sterilisation process caused an increase in MP release that ranged from $35 \%$ to $84 \%$ for the tested IFB products (Fig. 4b).

To assess the influence of repeated use of IFBs on MPs release, we conducted a 21-day test using $\mathrm{Pd} 1, \mathrm{Pd} 2$ and $\mathrm{Pd} 3$. The 21-day test showed that the MPs released from all three tested products had periodic fluctuations (Fig. 4c). This behaviour is likely due to the multilayer structure of ordered crystalline and amorphous layers in $\mathrm{PP}^{28}$ and is consistent with the flake-like multilayer appearance of the MPs released from IFBs observed using AFM (Fig. 3ik). The gradual degradation of the ordered crystalline structure results in the exposure of amorphous regions, which are susceptible to rapid degradation ${ }^{29}$. The detailed release mechanism requires further investigation. Although the fluctuations in Fig. 4c are significant, the lowest level of MP release was still higher than 640,000 particles/L.

Global infant exposure to MPs from IFBs. Given the widespread use of PP-IFBs (Fig. 1) and the large quantity of MPs released, we estimated the exposure of 12-month-old infants to MPs in 48 regions by using MP release rates from PP-IFBs measured in this work, the market share of each PP-IFB product, the non-breastfeeding rates and the infant daily milk-intake volume. We found that the overall average daily consumption of PP-MPs by infants per capita was 1,580,000 particles, with a range of 14,600 $-4,550,000$ particles, depending on regions (Fig. 5a, for calculation details see supplementary Note 7). This average level corresponds to about 2600 times the total adult consumption of MPs from water, food and air (up to 600 particles/day of adults ${ }^{10}$ ). Infants in Africa and Asia have the lowest potential exposure to MPs from IFBs, with rates of 527,000 particles/day and 893,000 particles/day, respectively. Infants in South America have medium exposure levels $(1,010,000$ particles/day) whereas Oceania, North American and Europe, have the highest level corresponding to 2,100,000 particles/day, 2,280,000 particles/day and 2,610,000 particles/day, respectively. We note, however, that there are inevitable uncertainties in the MPs exposure levels due to limitations in the available data and regional and time dependent variations of e-commerce business market share.

The striking contrast in the exposure rates between regions is related to differences in the breastfeeding rates and local preference for PP and non-PP products (Fig. 1 and Supplementary Tables 5 and 6$)$. Figure $5 b$ shows a robust linear correlation $\left(R^{2}=0.67\right.$, $\mathrm{P}<0.001$, indirect correlation) between the daily exposure to MPs and the breastfeeding rate. The significant differences in MP exposure levels in developed and developing regions is consistent with the well-established $10 \%$ reduction in breastfeeding rate for each doubling 
in the per capita gross domestic product ${ }^{30}$. In regions with similar breastfeeding rates, differences in the average exposure rates can be traced to a local preference for a specific IFB product. These data also suggest that with further economic development the decrease of the breastfeeding rate and the increase in formula consumption per infant ${ }^{30,31}$ will likely lead to an increased exposure of infants to MPs unless effective measures are taken to reduce the use of plastic products for infant feeding.

The transition from breastfeeding to bottle feeding depends on regional practices. Since the exposure to MPs may start at the earliest stages of postnatal development, we estimated the average MP consumption per infant in five countries during the first twelve months of life based on the limited data available (Fig. $5 c$ and Supplementary Table 8). During the first day following birth, an infant may consume up to 430,000 particles and for developed countries the consumption of MPs continues to increase month-on-month, reaching a maximum in or around months 5-6 as appetite levels increase. By months 6-7, the MP consumption level decreases due to reduced bottle formula consumption as complimentary foods are introduced into the diet. However, towards the end of the first year, the exposure to MPs increases once again as the cohort of infants that are breast-fed transition to being bottle-fed, reflecting in part the transition of breast-feeding mothers back to the workplace.

\section{Discussion}

We show that PP-IFBs are a source of infant MP exposure, yielding average exposure levels in excess of 1 million MPs per day, with generally higher rates in developed regions. These exposure levels are likely to be an underestimate - nano-sized particles (shown in Fig. $3 f-h$ ) are not captured in our measurements due to the use of $0.8 \mu \mathrm{m}$ pore size filters. Additionally, nano-plastics (NPs) tend to aggregate in water, making it challenging to accurately quantify NPs levels. Nevertheless, we found trillions of NPs per liter with a mean particle diameter of $\sim 100 \mathrm{~nm}$ (Supplementary Figure 8 and Note 8). Unaccounted sources of MP release from other PP products involved in formula preparation can also contribute to MP exposure. For example, electrical kettles used to heat water for formula preparation are commonly PPbased and account for $60 \%{ }^{32}$ and $91 \%$ (data mining from Amazon UK) of the EU and UK kettle markets, respectively. Tests involving 3 kettles from different manufacturers in the UK (Supplementary Figure 1a), revealed that over 10 million PP-MPs per litre are released into the water during a single boil, which is in line with previous findings ${ }^{33}$. The actual MP concentration in the prepared formula may be higher still, if the kettle used is not rinsed before each preparation and MPs are allowed to build up within it.

Given the strong dependence on water temperature (Fig. 4a), recommended and regional formula preparation procedures may lead to enhanced levels of MP release. We found that sterilisation, which is recommended by the $\mathrm{WHO}^{34}$ to eliminate bacteria from IFBs, exacerbates MP release. In practice, it may be possible to mitigate against the undesirable consequences of sterilisation by introducing an additional washing step using room temperature sterilised water (i.e., boiled water using non-PP kettles and cooled down to room temperature) to rinse away loose MPs present on the walls of the sterilised IFB. In contrast, the $\mathrm{WHO}^{34}$ and the UK Food Standards Agency ${ }^{35}$ recommend the water temperature for formula preparation be no less than $70^{\circ} \mathrm{C}$ to reduce the risks of exposure to Enterobacter sakazakii and Salmonella enterica in powder formula, which have been associated with severe illness and even deaths in infants ${ }^{34,36}$. In practice, however, only $71 \%$ 
of UK parents followed this guideline; $26 \%$ use boiled water ${ }^{37}$ - likely increasing the level of MP release. Additionally, we found that microwave heating can substantially increase MP levels due to the generation of localised pockets of superheated water (Supplementary Figure $1 \mathrm{~b}$ ). In practice, $35 \%$ of US parents of $0-9$ months old infants occasionally use a microwave to prepare powder formula, while around $20 \%$ of parents frequently prepare formula using this method ${ }^{38}$. The WHO strongly recommends against microwave use to avoid uneven heating ${ }^{26}$ - however, the additional unforeseen impact of PP becoming exposed to local pockets of superheated water results in higher levels of MP generation (Supplementary Figure 1b).

To date, there is no evidence for the presence of MPs in breast milk. Several nano-sized metal and inorganic particles, such as $\mathrm{Ag}^{39,40}$ and $\mathrm{TiO}_{2}{ }^{41-43}$, are known to pass through the mammary glands but further studies are needed to assess whether micro- and nano-sized plastic particles are expressed in human milk. However, our findings suggest that greater care may be needed in the handling of breast milk. Most breast pump-kits and feeding bottles are PP-based and risk the generation of MPs during cleaning and sterilisation. In the US, reheating of previously pumped breast milk is common practice among breast feeding mothers ( $>80 \%$ of 2 -month-old infants' mothers), $12 \%$ of whom reheat the milk using a microwave oven ${ }^{38}$. Therefore, while the levels of exposure to MPs is significantly reduced for breast-fed infants, there are still potential risks that can be mitigated against by changing local practices.

Given the global preference for PP-IBFs, it is important to mitigate against the unintended generation of micro- and nano-plastics in infant formula by, for example, preparing sterilised water using non-PP cookware. However, the prevalence of plastic products in daily food storage and food preparation, and the fact that every PP product tested in our study (infant bottles, kettles, lunch boxes and noodle cups) released similar levels of MPs, points to an urgent need for technological solutions. The degradation of PP in contact with water suggests that surface coatings, functionalisations and plasma treatments may be helpful in reducing or preventing MP generation. However, the development of effective mitigation strategies and policies to ensure the safe use of plastics must be based on known risk levels, which have yet to be established. There must now be an immediate focus to assess the potential risk of micro- and nano-plastics to human health and to develop plastic fabrication technologies that yield products that are not easily degraded by water or the environment.

\section{Methods}

Precautions to prevent sample contamination. To avoid potential MP contamination, all hardware that came in contact with the samples were made from clean glass. During the sample preparation and test process, particle-free nitrile gloves and laboratory coats (100\% cotton) were worn. The sleeves of coats were inserted and fixed inside of the nitrile gloves. Before every work step, the gloves were thoroughly washed by distilled water, followed by deionized (DI) water. A blank control sample was analyzed with every 10 PP-product samples to determine the background MPs concentration in DI water. Prior to sample preparation, we highlight 3 processes that in our experience must be avoided due to the potential for MP contamination: (1) Sonicating of IFBs as part of the cleaning process, (2) Heating DI water using plastic kettles, and (3) Directly heating waterfilled PP products using a microwave (Details in Supplementary Notes 1-2).

Identification and characterization of PP-MPs. According to an established ${ }^{16,17,44}$ and independently validated test protocol (Supplementary Notes 3-4), Raman spectroscopy (Renishaw InVia Raman spectrometer) was used to identify the presence of MPs in the samples prepared as the description in Fig. 2a. MPs particles released 
from PP products onto the surface of filter membranes were identified by using a Renishaw InVia Raman spectrometer. The WiRE 3.4 (Renishaw) software was used and the system was equipped with a $532 \mathrm{~nm}$ laser (Coherent Inc.), a cooled charge-coupled device (CCD) and a microscope (NT-MDT) with a 100x objective (Mitutoyo, M Plan Apo, N.A.=7.0). Before every test, a system calibration was conducted using a silicon wafer. During testing, a low excitation laser power $(\sim 180 \mu \mathrm{W})$ was applied on the sample, which provided clear spectra of MPs without damaging the MPs particles under high laser power density. The exposure time was set to 20 seconds with accumulations of 10-40 times depending on the intensity of the Raman signal. Intense Raman peaks in the range of $2830-2970 \mathrm{~cm}^{-1}$ (stretching vibrations of $\mathrm{CH} / \mathrm{CH}_{2} / \mathrm{CH}_{3}$ groups) were used to determine the PP-MPs in this study. The quantity and size of the PP-MPs were analysed using ImageJ. More details are shown in Supplementary Notes 2-3. To obtain three-dimensional topography of PP-MPs, an NT-MDT AFM (AFM, operating with a Nova NT-MDT SPM software) was employed. Prior to testing, 2-3 drops of the water sample from the IFB were dropped and dried on the surface of a clean silica wafer. Then the sample was tested with a tapping mode probe (Nanosensors, PPP-NCST) (details in Supplementary Note 6).

Control experiment to check the influence of infant formula, tap water and DI water on PP release from IFBs. To compare MP release levels between DI water and tap water, the DI water sample was prepared using the method described in Fig. 2a and Supplementary Note 2.2. The tap-water sample was prepared by replacing DI water with tap water. When testing for MPs release from IFBs containing infant formula, we found that the presence of large quantities of formula particles hampered quantitative testing even though Raman was easily able to distinguish between formula and PP-MPs particles (Fig. $2 \mathrm{~h}$ ). We modified the procedure to assess whether formula affects the release of PP-MPs particles due to, for example, the formation of a passivating film on the inner surface of IFBs (experiment details in Supplementary Figure 7 and Note 5.2). A statistical analysis involving a two-sample student's $t$-test (two-sided) was conducted (OriginPro 8.6) to determine if PPMPs results obtained from different conditions and labs are significantly different. The null hypothesis $\left(H_{0}\right)$ is that there is no difference between the two sets of samples. With a confidence level of $95 \%$, a $P$ value $<0.05$ was assumed to be significant (rejecting $\mathrm{H}_{0}$ ). At the statistical analysis of third-party and our lab results, the degrees of freedom and $P$ value were 8 and 0.66 , respectively. At the analysis of tap water and DI water, the degrees of freedom and $P$ value obtained using $P d 1$ and $P d 2$ were $8,0.13$ and 8, 0.18, respectively. At the analysis of real formula and DI water, the degrees of freedom and P value obtained using Pd1 and Pd 2 were 8 , 0.89 and $8,0.96$, respectively. The results that the MP levels released by DI water alone are not significantly different $(P>0.05)$ from IFBs that had a residual coating of infant formula mixture on the inner surface, regardless of the product used (Fig. $2 \mathrm{i}$ ). These results indicate that infant formula does not form a protective film on the inner surface of IFBs that prevents MP release and confirm that different water types (tap/DI) did not significantly change the quantity of MPs released.

MPs release from PP-IFBs in different daily-use scenarios. To investigate the MP release characteristics of 10 representative PP-IFBs products (Pd1-Pd10), we carefully followed the user instructions for the IFBs recommended by $\mathrm{WHO}$ and the sample preparation involved 4 steps: clean/sterilisation, air dry, mixing and filtering (details shown in Supplementary Note 2). To assess the influence of temperature on MP release, we thoroughly cleaned and air-dried 20 PP-IFBs for each of three IFB products Pd1, Pd2 and Pd3. DI water with temperatures of $25^{\circ} \mathrm{C}, 40^{\circ} \mathrm{C}, 70^{\circ} \mathrm{C}$ and $95^{\circ} \mathrm{C}$ was poured into the IFBs (5 IFBs from each product experienced the same temperature treatment), and then shaken for 60 s and the water samples were tested for MPs as described in Supplementary Note 2.3 . Since $100^{\circ} \mathrm{C}$ sterilisation is a standard cleaning procedure for IFBs (a WHO recommendation), we also compared the MP released from IFBs with and without sterilisation (Supplementary Note 2.3) followed by exposure to $70^{\circ} \mathrm{C}$ water (the temperature recommended by WHO for infant formula preparation) using the protocol described in Supplementary Note 2.2. To assess the influence of repeated use of IFBs on MPs release, we conducted a 21-day test using Pd1, Pd2 and Pd3 (5 samples of each product). During testing, the bottles were sterilised once a day and used for formula preparation 5 times per day (Supplementary Note 2.4).

Assessment of infant's MP exposure due to PP-IFB use worldwide. We estimated the infants exposure to MPs in 48 regions by using MP release rates from PP-IFBs (data from this study), the market shares of PP-IFBs (Supplementary Tables 5 and 6), the non-breastfeeding rates (data from the Lancet ${ }^{30}$ and Government reports, Supplementary Table 5) and the infant daily milk-intake volume (Supplementary Table 8). For each region, the MPs exposure was assessed according to equations 1-3. 


$$
\begin{gathered}
M P_{i}=\left(1-B F R_{i}\right) \times P_{P P} \times A_{\text {avg }} \times V_{i} \\
A_{\text {avg }}=\frac{\sum_{i=1}^{10} \sum_{j=1}^{3} A_{i j} P_{i j}}{\mathrm{P}_{P d 10}} \\
\mathrm{P}_{P d 10}=\sum_{i=1}^{10} \sum_{j=1}^{3} P_{i j}
\end{gathered}
$$

Where $M P_{i}, B F R_{i}, V_{i}$ is the daily MPs exposure, the breastfeeding rate and the daily milk-intake volume of $i$ month-old infants in each region, respectively. $P_{P P}$ is the market share of PP related IFB products in each region. $A_{\text {avg }}$ is the overall average MPs amount released from PP related IFB products in each region. $P_{P d 10}$ is the overall market share of the selected 10 PP-IFB products in each region. Index $i$ refers to the products (Pd 1 to $\mathrm{Pd} 10$ ), whereas the index $j$ refers to the PP form factor ( $j=1$ bottle only; $j=2$ both bottle and accessories; $j=3$ accessories only). For example, $A_{11}$ is the quantity of MPs released from the PP bottle $(j=1)$ from Pd $1(i=1)$. The data of $A_{i j}$ were obtained by experiment while IFBs sales details were obtained from data mining of local leading e-commerce sites in each region (Supplementary Table 5). Additional details on the assessment method is provided in Supplementary Note 7.

\section{Data availability}

All data analysed in this study is contained within the Supplementary Information. The raw data that support the findings of this study are available from the corresponding author upon request to JJW or from Figshare repository at https://figshare.com/account/home\#/projects/88406.

\section{Code availability}

The mathematical algorithm used for MPs exposure assessment has been included in the Method section. The sales data mining software-Jungle scout is available at https://www.junglescout.com/.

\section{References}

1 Thompson, R. C. et al. Lost at sea: where is all the plastic? Science 304, 838 (2004).

2 Efferth, T. \& Paul, N. W. Threats to human health by great ocean garbage patches. The Lancet Planetary health 1, e301-e303 (2017).

3 Gupta, J. et al. Communicating the health of the planet and its links to human health. The Lancet Planetary health 3, e204-e206 (2019).

4 de Sá, L. C., Oliveira, M., Ribeiro, F., Rocha, T. L. \& Futter, M. N. Studies of the effects of microplastics on aquatic organisms: what do we know and where should we focus our efforts in the future? Science of the total environment 645, 1029-1039 (2018).

5 Wright, S. L. \& Kelly, F. J. Plastic and human health: a micro issue? Environmental science \& technology 51, 6634-6647 (2017).

6 Meissner, R. Ocean governance for human health and the role of the social sciences. The Lancet Planetary health 2, e275-e276 (2018).

7 The, L. P. H. Microplastics and human health-an urgent problem. The Lancet Planetary health 1, e254 (2017).

8 Smith, M., Love, D. C., Rochman, C. M. \& Neff, R. A. Microplastics in seafood and the implications for human health. Current environmental health reports 5, 375-386 (2018). 

the environment and possible impact on human health. Environmental science \& technology 53, 1748-1765 (2019).

Cox, K. D. et al. Human consumption of microplastics. Environmental science \& technology 53, 7068-7074, doi:10.1021/acs.est.9b01517 (2019).

11 Schwabl, P. et al. Detection of various microplastics in human stool: a prospective case Series. Annals of internal medicine 171, 453-457 (2019).

12 Jin, Y., Lu, L., Tu, W., Luo, T. \& Fu, Z. Impacts of polystyrene microplastic on the gut barrier, microbiota and metabolism of mice. Science of the total environment 649, 308-317 (2019). Lu, L., Wan, Z., Luo, T., Fu, Z. \& Jin, Y. Polystyrene microplastics induce gut microbiota dysbiosis and hepatic lipid metabolism disorder in mice. Science of the total environment 631, 449-458 (2018).

14 Mattsson, K. et al. Brain damage and behavioural disorders in fish induced by plastic nanoparticles delivered through the food chain. Scientific reports 7, 11452 (2017).

15 World Health Organization. Microplastics in drinking-water. (2019).

16 Oßmann, B. E. et al. Small-sized microplastics and pigmented particles in bottled mineral water. Water research 141, 307-316 (2018).

17 Schymanski, D., Goldbeck, C., Humpf, H.-U. \& Fürst, P. Analysis of microplastics in water by micro-Raman spectroscopy: release of plastic particles from different packaging into mineral water. Water research 129, 154-162 (2018).

18 Hernandez, L. M. et al. Plastic teabags release billions of microparticles and nanoparticles into tea. Environmental science \& technology 53, 12300-12310 (2019).

19 Geyer, R., Jambeck, J. R. \& Law, K. L. Production, use, and fate of all plastics ever made. Science advances 3, e1700782 (2017).

20 Tripathi, D. Practical guide to polypropylene (Rapra Technology, Shrewsbury, 2002).

21 Zimmermann, L., Dierkes, G., Ternes, T. A., Völker, C. \& Wagner, M. Benchmarking the in vitro toxicity and chemical composition of plastic consumer products. Environmental science \& technology 53, 11467-11477 (2019).

22 Zhao, S. et al. Analysis of suspended microplastics in the Changjiang Estuary: Implications for riverine plastic load to the ocean. Water research 161, 560-569 (2019).

23 Pan, Z., Liu, Q., Sun, Y., Sun, X. \& Lin, H. Environmental implications of microplastic pollution in the Northwestern Pacific Ocean. Marine pollution bulletin 146, 215-224 (2019).

24 Efimova, I., Bagaeva, M., Bagaev, A., Kileso, A. \& Chubarenko, I. P. Secondary microplastics generation in the sea swash zone with coarse bottom sediments: Laboratory experiments. Frontiers in Marine Science 5, 313 (2018).

25 Klein, S., Dimzon, I. K., Eubeler, J. \& Knepper, T. P. in Freshwater microplastics-Emerging Environmental Contaminants? 51-67 (Springer, Cham, 2018).

26 World Health Organization. How to prepare formula for bottle-feeding at home (2007).

27 Zhao, S., Danley, M., Ward, J. E., Li, D. \& Mincer, T. J. An approach for extraction, characterization and quantitation of microplastic in natural marine snow using Raman microscopy. Analytical methods 9, 1470-1478 (2017).

28 Simpson, R. J. \& Selke, S. E. in Emerging Technologies in Plastics Recycling (ed Gerald D. Andrews) Ch. 18, 232-240 (ACS Publications, 1992).

29 Longo, C., Savaris, M., Zeni, M., Brandalise, R. N. \& Grisa, A. M. C. Degradation study of polypropylene (PP) and bioriented polypropylene (BOPP) in the environment. Materials research 14, 442-448 (2011).

30 Victora, C. G. et al. Breastfeeding in the 21st century: epidemiology, mechanisms, and lifelong effect. The Lancet 387, 475-490 (2016).

31 Neves, P. A. et al. Infant formula consumption is positively correlated with wealth, within and between countries: a multi-country study. The Journal of nutrition 150, 910-917 (2020). 
32 Gallego-Schmid, A., Jeswani, H. K., Mendoza, J. M. F. \& Azapagic, A. Life cycle environmental evaluation of kettles: Recommendations for the development of eco-design regulations in the European Union. Science of the total environment 625, 135-146 (2018).

33 Sturm, M. T., Kluczka, S., Wilde, A. \& Schuhen, K. Determination of particles produced during boiling in differenz plastic and glass kettles via comparative dynamic image analysis using FlowCam ${ }^{\circledR}$. (2019).

34 World Health Organization. Safe preparation, storage and handling of powdered infant formula: guidelines. (2007).

35 Food Standards Agency and Department of Health. Guidance for health professionals on safe preparation, storage and handling of powdered infant formula. (2005).

36 Angulo, F. J., Cahill, S. M., Wachsmuth, I. K., Costarrica, M. d. L. \& Embarek, P. K. B. Powdered infant formula as a source of Salmonella infection in infants. Clinical infectious diseases 46, 268-273 (2008).

37 National Health Service, UK. Infant Feeding Survey - UK, 2010. (NHS Digital, 2012).

38 Centers for Disease Control and Prevention, US. Breastfeeding Among U.S. Children Born 2009-2016, CDC National Immunization Survey. (2019).

39 Morishita, Y. et al. Distribution of silver nanoparticles to breast milk and their biological effects on breast-fed offspring mice. ACS nano 10, 8180-8191 (2016).

40 Melnik, E. et al. Transfer of silver nanoparticles through the placenta and breast milk during in vivo experiments on rats. Acta Naturae 5 (2013).

41 Gao, X. et al. Effects of developmental exposure to TiO 2 nanoparticles on synaptic plasticity in hippocampal dentate gyrus area: an in vivo study in anesthetized rats. Biological trace element research 143, 1616-1628 (2011).

42 Zhang, C. et al. Induction of size-dependent breakdown of blood-milk barrier in lactating mice by TiO2 nanoparticles. PloS one 10 (2015).

43 Cai, J., Zang, X., Wu, Z., Liu, J. \& Wang, D. Translocation of transition metal oxide nanoparticles to breast milk and offspring: The necessity of bridging mother-offspringintegration toxicological assessments. Environment international 133, 105153 (2019).

44 Oßmann, B. E. et al. Development of an optimal filter substrate for the identification of small microplastic particles in food by micro-Raman spectroscopy. Analytical and bioanalytical chemistry 409, 4099-4109 (2017).

\section{Acknowledgements}

This work was supported by Enterprise Ireland (grant number CF20180870); Science Foundation Ireland (grants numbers: 12/RC/2278 and 16/IA/4462); School of Engineering Scholarship at Trinity College Dublin; China Scholarship Council (201506210089 and 201608300005). We also thank Keyence for their help.

\section{Author contributions}

JJW and LX supervised the project and led the overall effort. DL, JJW, JJB and LX wrote the manuscript. JJB contributed to experimental design and validation. YS and DL performance online data mining and analysing. DL, YS and LY carried out the sample preparation. DL and JJW carried out the Raman measurements. DL, DKK and JJW carried out AFM and SEM characterization. All authors discussed the results and commented on the manuscript.

\section{Competing interests}

The authors declare no competing interests. The presentation of the material in this publication does not imply the expression of any opinion whatsoever on the part of Trinity College Dublin about specific companies or of certain manufacturers' products and does not imply that they are endorsed, recommended, criticised or otherwise by Trinity College Dublin in preference to others of a similar nature. Errors and omissions excepted. 
All reasonable precautions have been taken to verify the information contained in this publication. However, the published material is being distributed without warranty of any kind, either expressed or implied. The responsibility for the interpretation and use of the material lies with the reader. In no event shall Trinity College Dublin be liable for damages arising from its use.

\section{Supplementary Information}

Supplementary Figures $1-8$

Supplementary Tables $1-8$

Supplementary Notes 1 - 8

\section{Figure}

a

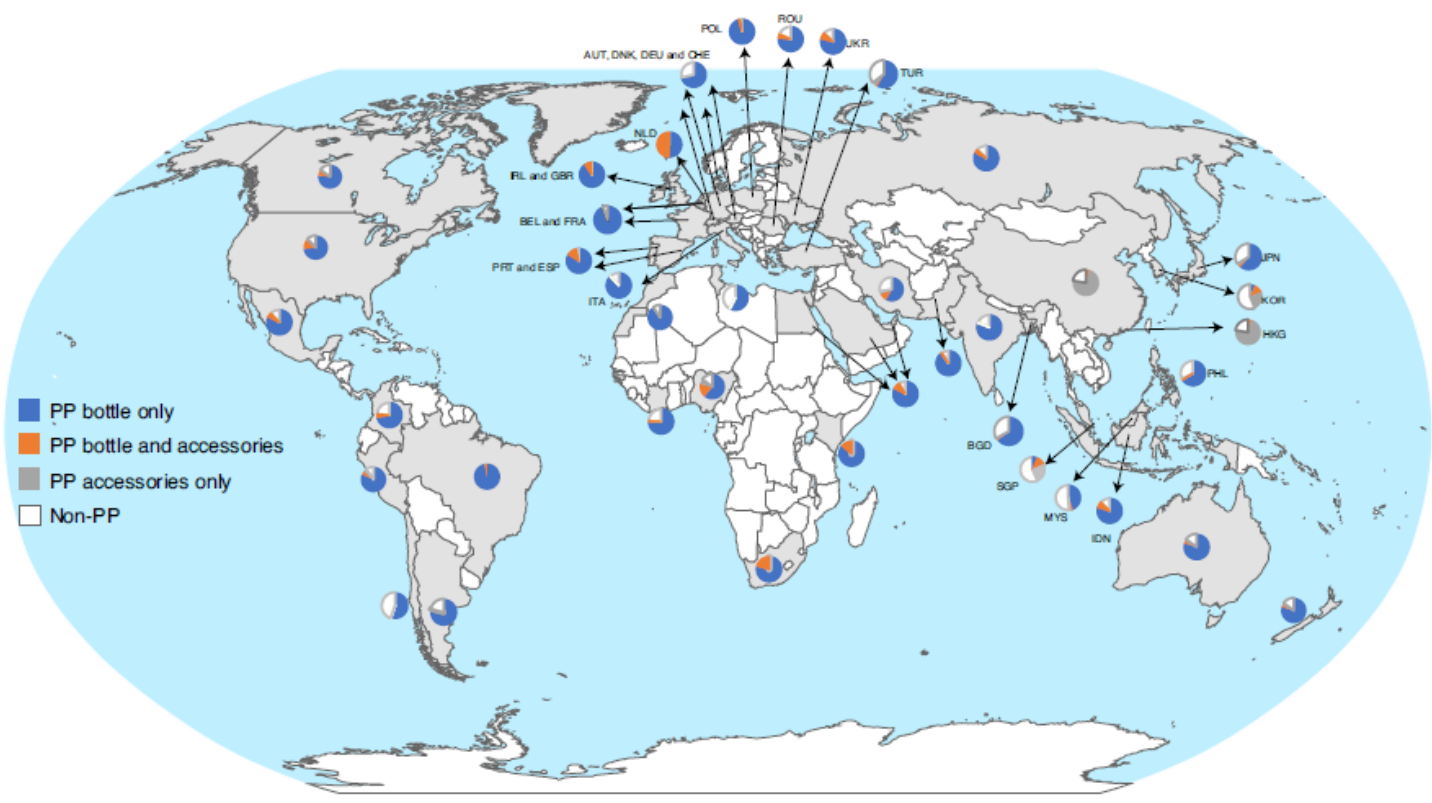

b

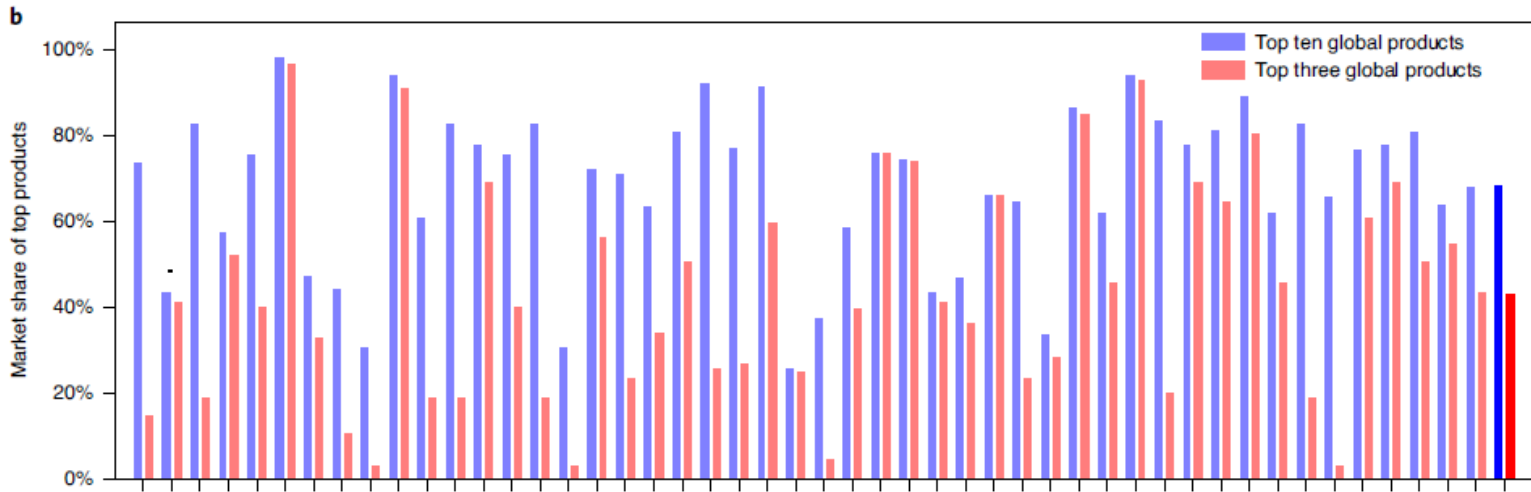

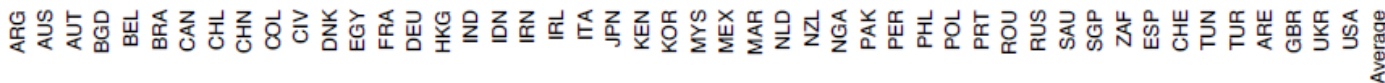

Fig. 1 | The world market share of PP-IFBs. (a) Regional breakdown of IFB e-commerce sales across four categories. (PP-Bottle only: only the IFB body is made of PP but without PP accessories; PP-Bottle \& Accessories: both the IFB body and accessories are made of PP; PP-Accessories only: includes only IFB accessories (without a PP-bottle) that are made of PP, such as PP straws and gravity balls, that are placed inside of the non-PP IFB body. Note these accessories come into contact with the formula during preparation; Non-PP: neither the IFB body or the accessories are composed of PP, e.g., glass IFBs without PP accessories). (b) The local market shares of the global top 3 and top 10 IFB products in each of the $\mathbf{4 8}$ regions. For data mining details see Supplementary Tables 5 and 6. 


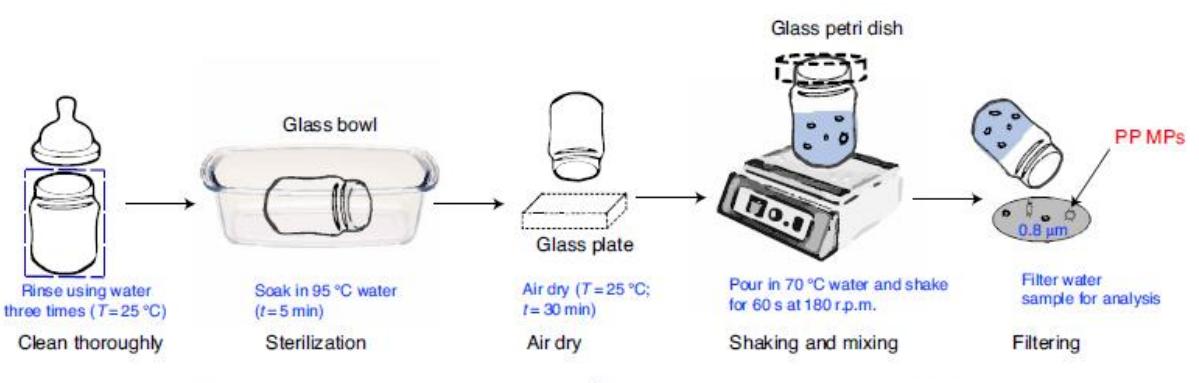

b

c
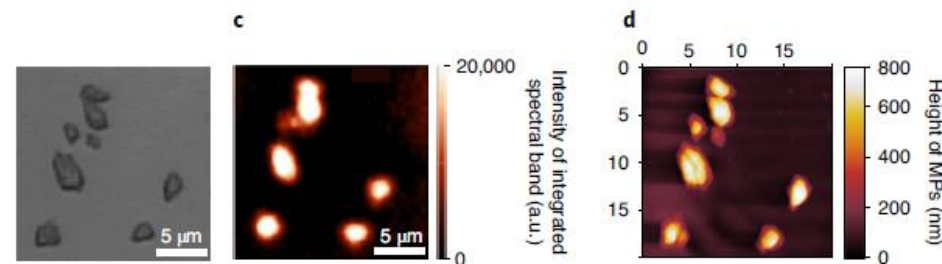

e
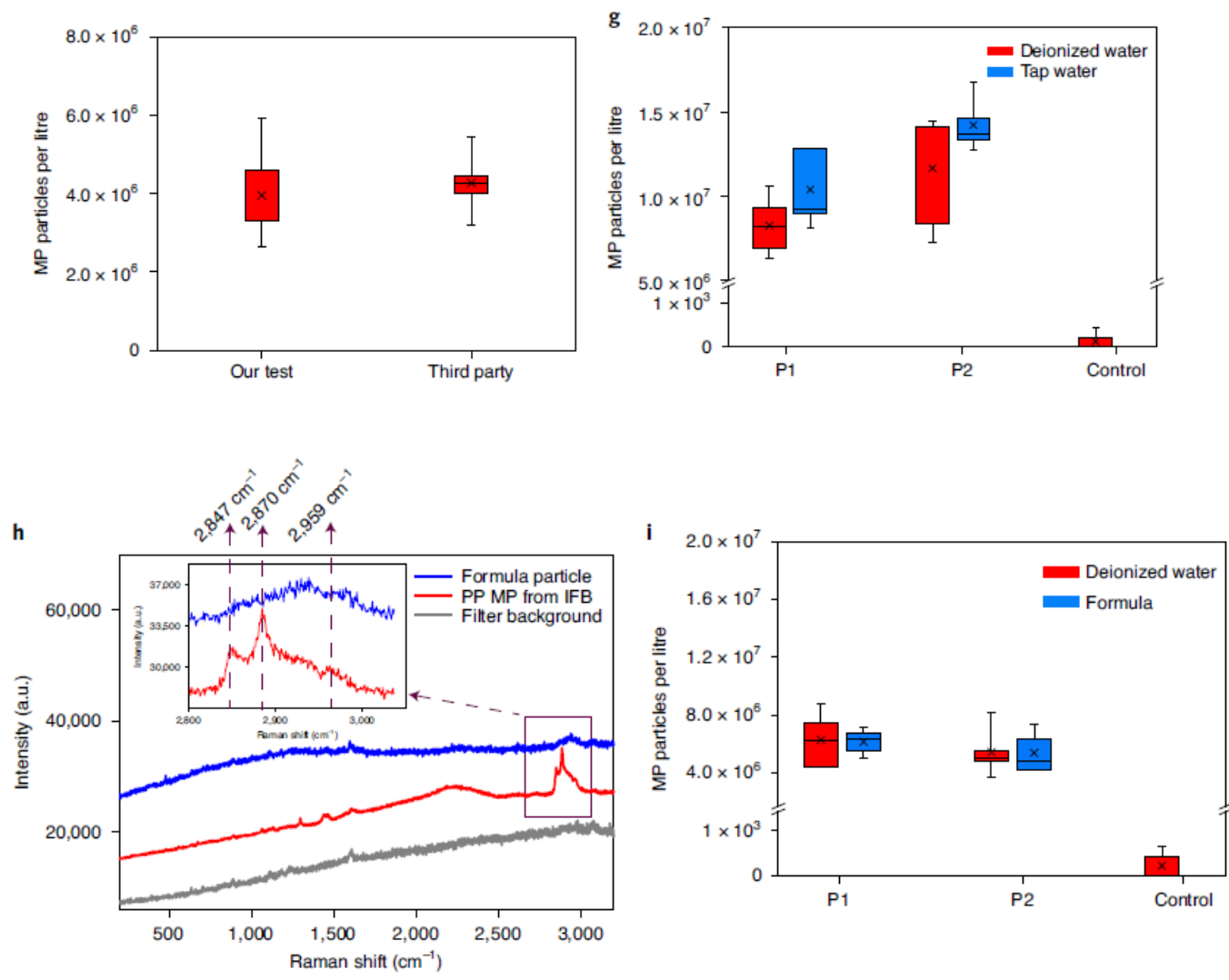

Fig. 2 | IFBs sample preparation and the establishment and validation of MPs analysis protocol. (a) Schematic diagram of IFBs sample preparation. (b-e) Identification and mapping of PP-MPs: (b) Optical microscopic image of particles released from IFBs with 100x microscope objective; (c) The Raman mapping of the same region obtained using the PP Raman bands at $2830-2870 \mathrm{~cm}^{-1}$. The color scale bar indicates the intensity of the integrated spectral band in arbitrary units; (d) The AFM image of the same region to determine the morphology of released MPs. The color scale bar indicates the height of MPs; (e) 3D AFM topographic image. (f) The results obtained from the third party and our lab, respectively. IFB product $1(\mathrm{Pd} 1)$ was used in this test. $(n=5$ independent bottles used for each lab test, $p=0.66$ using two-sided $t$ test with a confidence interval of $95 \%$, the same statistical analysis was used in the following significance analysis, details see method). (g) MPs released from PP-IFBs using tap water and DI water, respectively. For the control samples, there was no PPMPs found when using tap water ( $\mathrm{n}=5$ independent bottles of Pd1 and product $2(\mathrm{Pd} 2)$ in both tap water and DI water tests, respectively. The $P$ values obtained using $P d 1$ and $P d 2$ were 0.13 and 0.18 , respectively). (h) The Raman spectra of PP-MPs, formula particles and filter background. (i) MPs released from PP-IFBs using formula and DI water. For the control samples, the PP-IFBs were replaced by a glass beaker and the same 
procedure was followed for formula and DI water sample preparation, respectively. For the control samples, there was no PP-MPs found when using formula while only very small amounts of MPs were found in DI water control samples, which is most likely due to the plastic tubing used in DI water making process $(n=5$ independent bottles of $\mathrm{Pd} 1$ and $\mathrm{Pd} 2$ used in formula and $\mathrm{DI}$ water tests, respectively. The P values obtained using $\mathrm{Pd} 1$ and $\mathrm{Pd} 2$ were 0.89 and 0.96 , respectively). 


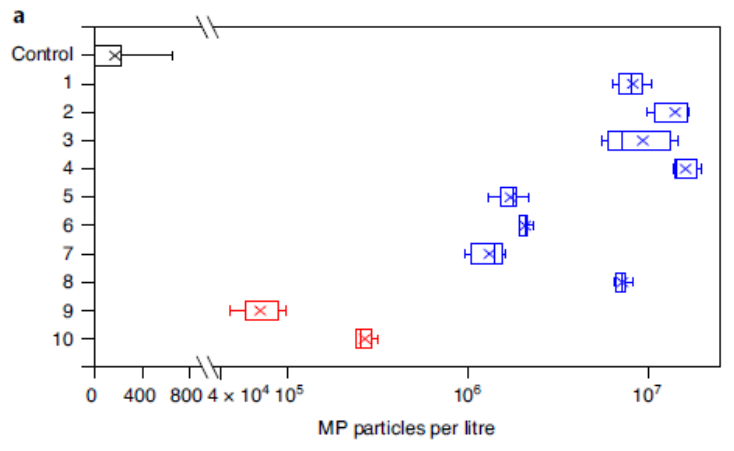

c 0
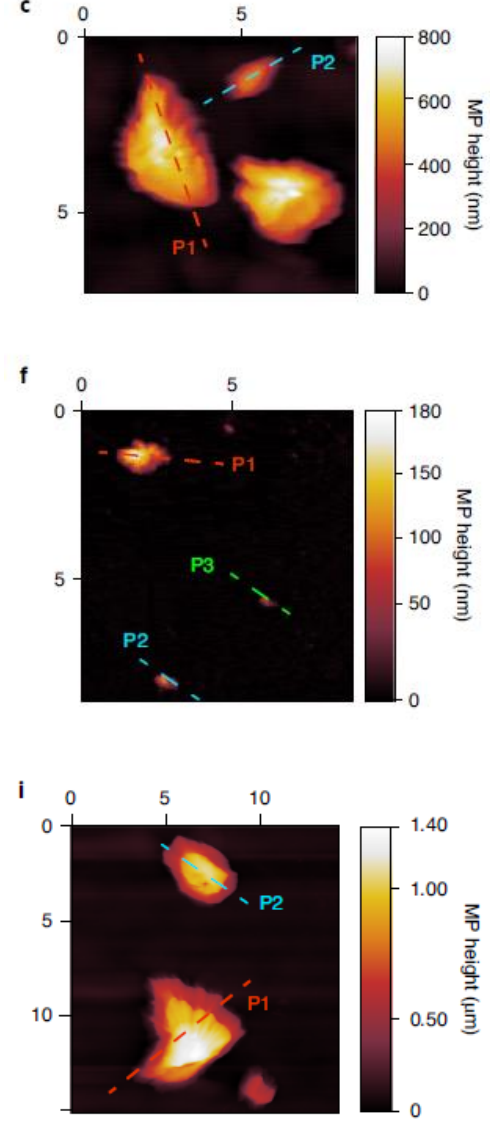
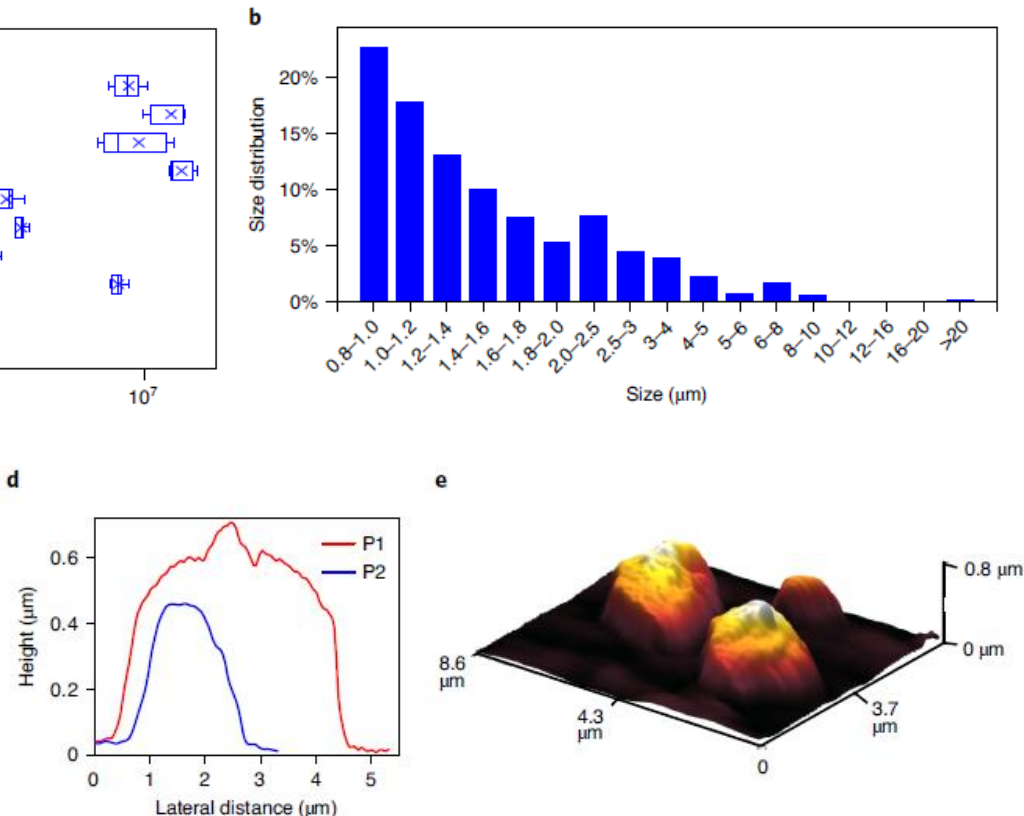

h
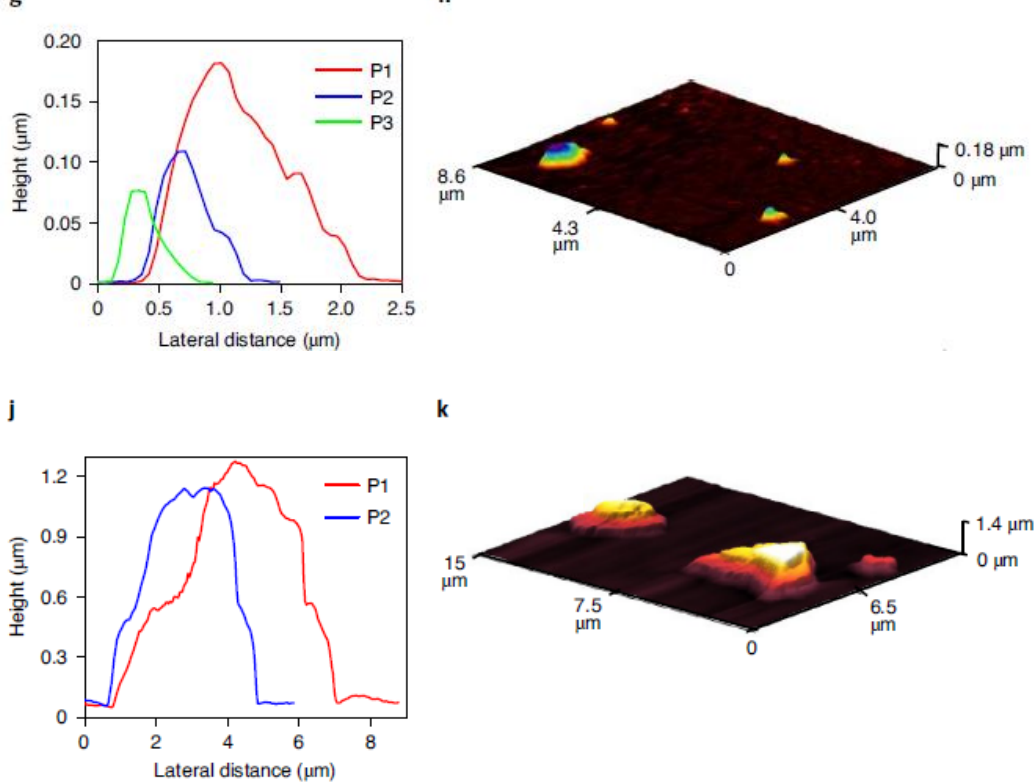

Fig. 3 | The MPs released from PP-IFBs. (a-b)The MPs released from Products 1-10 and the MP size distribution (a) Quantity of MPs released from PP-IFBs Products 1-10 (Pd=Product, e.g., Pd1-Product 1; Ctrlcontrol samples, the preparation of the control and IFB samples are described in Supplementary Note $1 ; n=5$ independent samples were used to test each product, $n=18$ independent control samples were used). The individual contributions to each product (bottle only, bottle and accessories and accessories only) are detailed at Supplementary Table 1. (b) Typical size distribution of MPs released from PP-IFB Pd1. The size distribution for Pd2-10 is shown in Supplementary Figure 2. (c-k) Topographic maps of MPs released from PP-IFBs. (c), (d) and (e) showed the AFM image, extracted cross section profile and 3D topographic image of released MPs with sizes from 2-5 $\mu \mathrm{m}$, respectively. (f), (g) and (h) showed examples of MPs with sizes less than $2 \mu \mathrm{m}$. (i), (j) and (k) showed that MPs typically exhibit a multi-layer structure. All MPs tested by AFM were previously identified by Raman spectroscopy. To highlight the 3D structure of the MPs the vertical height dimension is exaggerated; The color scale bar on the right of $\mathbf{c}, \mathbf{f}$ and $\mathbf{i}$ indicates the height of MPs. 

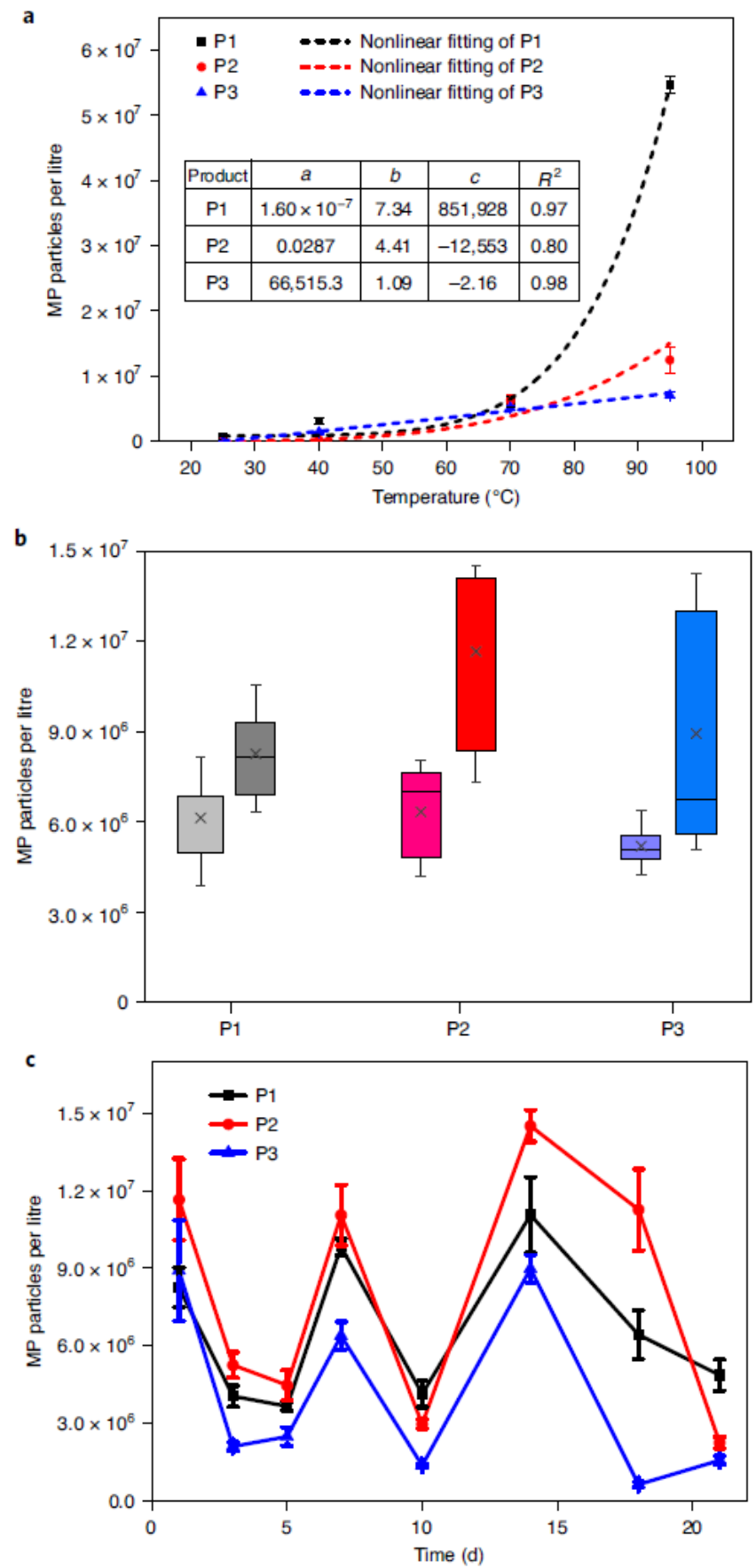

Fig. 4 I The impact of temperature and repeated-use on MPs release from three PP-IFB products (not including accessories). (a) The quantity of MPs released at different temperature, including a fit to these data. The power law equation (MPs=axTemp ${ }^{\mathrm{b}}+\mathrm{c}$ ) was used to fit the MPs release with temperature, the fit results are listed in the inset table ( $n=5$ independent bottles of each product were tested at each temperature). (b) The quantity of MPs released with and without the sterilisation. Sterilisation involved a 5 mins soak in $95^{\circ} \mathrm{C}$ hot water ( $n=5$ independent bottles of each product Pd1-3 were tested with and without the sterilisation, respectively). (c) The quantity of MPs released over a 21-day testing period (The sample was collected after sterilisation process, more details can be found in Supplementary Table 3; $n=5$ independent bottles for each tested product Pd1-3, respectively). All the error bars in Figs. a and c indicate the standard error of mean values. 


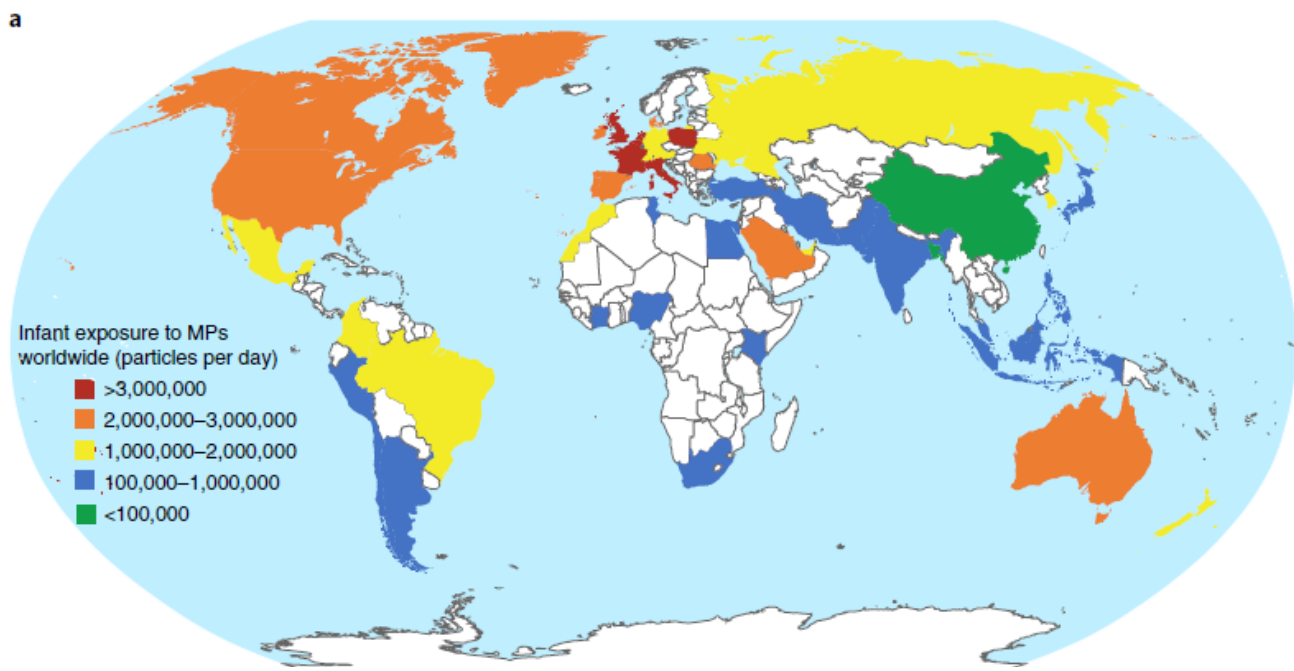

b
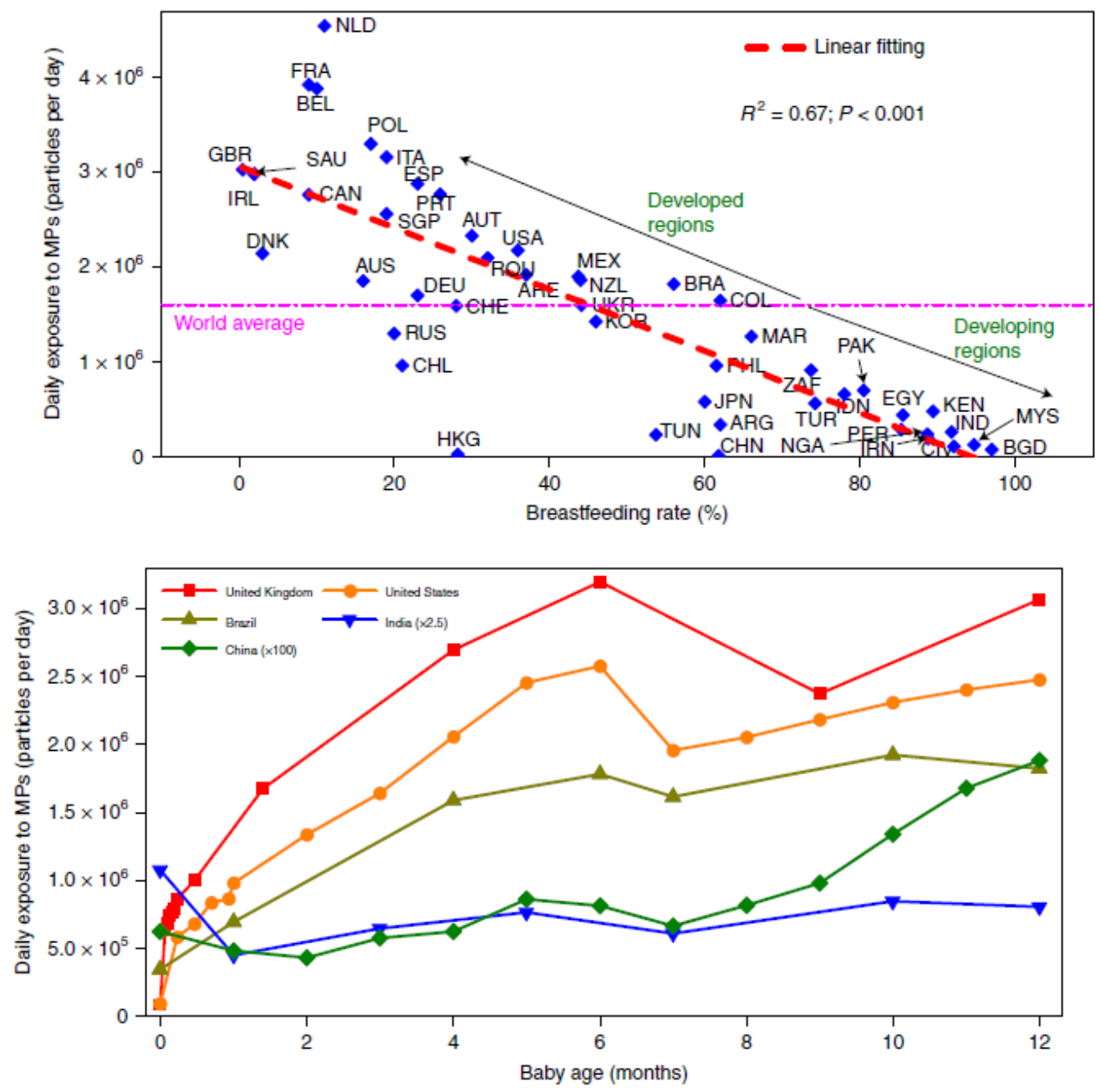

Fig. 5 | MP daily exposure to infants worldwide. (a) The world map of the daily exposure of 12-month-old infants to MPs due to the use of 10 PP-IFB products (Table S1). (b) Plot of the daily MP exposure at 12-months vs the local the breastfeeding rate (Alpha-3 code was used to indicate each region, e.g., IRL-Ireland; BRbreastfeeding rate). (c) The infants' MPs exposure during the first 12 months (Month 0 represent the first hour after birth) in five countries. The consumption level for India is $\times 2.5$ while China is $\times 100$ to illustrate the trend - China's low level reflects a local preference for non-PP body IFBs. 